\title{
Decreased GFR and its joint association with type 2 diabetes and hypertension with prevalence and severity of carotid plaque in a community population in China
}

This article was published in the following Dove Press journal: Diabetes, Metabolic Syndrome and Obesity: Targets and Therapy

\author{
Qianzi Che' \\ Ying Yang ${ }^{2}$ \\ Guanliang Cheng ${ }^{2}$ \\ Jia Jia ${ }^{2}$ \\ Fangfang Fan $^{2}$ \\ Jianping $\mathrm{Li}^{2}$ \\ Yong Huo ${ }^{2}$ \\ Dafang Chen' \\ Yan Zhang ${ }^{2}$ \\ 'Department of Epidemiology \& \\ Biostatistics, Peking University Health \\ Science Center, Beijing, People's Republic \\ of China; ${ }^{2}$ Department of Cardiology, \\ Peking University First Hospital, Beijing \\ People's Republic of China
}

Background: Type 2 diabetes (T2DM), hypertension and kidney dysfunction are known risk factors for cardiovascular disease, but their combined effect on carotid plaque remains uncertain. This study aims to assess the associations between T2DM, hypertension, kidney dysfunction and carotid plaque, and further explore the combined effect of three diseases.

Patients and methods: We conducted a cross-sectional analysis among 3,815 communitydwelling adults in a Chinese atherosclerosis cohort. Estimated glomerular filtration rate (eGFR), hypertension and T2DM were evaluated as risk factors for carotid plaque. The presence, number and total area of carotid plaques were also assessed. Using logistic model, mutinomial logistic model and generalized linear regression model, the relationship between risk factors and carotid plaque was examined.

Results: T2DM, hypertension, decreased GFR, and, inversely, eGFR, were independently associated with the presence, number and total area of carotid plaque. Stratified analysis by T2DM and hypertension showed T2DM attenuated the association between eGFR change and carotid plaque. There was a cumulative relationship between three risk factors and carotid plaque burden. The OR for the number of plaques was 1.0 (reference), 1.55 to 2.03, 1.94 to 3.14 , and 3.69 (all $P<0.05$ ), respectively, for individuals with none, one, two, and three risk factors. Likewise, combining three risk factors was associated with greater increase in total plaque area $(\beta, 20.63 ; 95 \% \mathrm{CI}, 14.04-27.22)$.

Conclusion: The coexistence of decreased GFR, diabetes and hypertension is associated with increased risk of carotid plaque, and these comorbidities may contribute additively to the development of plaque.

Keywords: joint association, decreased GFR, T2DM, hypertension, carotid plaque

Correspondence: Dafang Chen Department of Epidemiology and Biostatistics, Peking University Health Science Center, No. 38 Xueyuan Road, Haidian District, Beijing I0019I, People's

Republic of China

Tel +86 1082802644

Fax +86 1082802644

Email1308065I28@qq.com

Yan Zhang

Department of Cardiology, Peking

University First Hospital, 8 Xishiku Street,

Xicheng District, Beijing, People's

Republic of China

Tel +86 0l0-83575728

Email drzhyl108@I63.com

\section{Introduction}

Carotid plaque has been reported as a strong predictor of incident myocardial infarct and ischemic stroke, ${ }^{1-4}$ and helped reclassify up to $22.7 \%$ of participants at intermediate risk of coronary heart disease in the Atherosclerosis Risk in Communities study. ${ }^{5}$ Therefore, research on the risk factors for carotid plaque could aid in the prevention of cardiovascular disease (CVD).

Traditional vascular risk factors include age, sex, smoking, diabetes, hypertension, family history of MI, physical activity (inverse), statin use and antihypertensive medication. ${ }^{6}$ In addition to the established risk factors, accumulating evidence 
suggests that kidney dysfunction may also increase the risk of atherosclerosis. Investigators have now demonstrated a two-fold increase in the risk of developing atrial fibrillation, congestive heart failure, coronary heart disease, and stroke in patients with estimated glomerular filtration rate (eGFR) $<60 \mathrm{~mL} / \mathrm{min} / 1.73 \mathrm{~m}^{2} .^{7-10}$ However, the correlation between kidney dysfunction and carotid plaque remains uncertain. ${ }^{11}$

Type 2 diabetes (T2DM) and hypertension are currently the most common causes of chronic kidney dysfunction in developed countries. Moreover, hypertension increases the risk of renal and cardiovascular morbidity and mortality in diabetic patients. ${ }^{12-15}$ Therefore, it is imperative to recognize the excess risk of developing atherosclerosis in patients with these conditions. Although it is well established that T2DM and hypertension are most prevalent risk factors for atherosclerosis, ${ }^{16-19}$ the joint effect of T2DM, hypertension and kidney dysfunction on carotid plaque has not been previously assessed. Herein, we measured GFR within a Chinese community-based atherosclerosis cohort, which concurrently collected information on carotid atherosclerosis content from all study participants via carotid ultrasonography. Our objective was to assess the joint associations of kidney dysfunction, especially decreased GFR, and the established risk factors of T2DM and hypertension with carotid plaque.

\section{Materials and methods Study subjects}

This study used data from a community-based CVD cohort among residents in Gucheng and Pingguoyuan Community of Shijingshan District in Beijing, China, carried out from December 2011 to 2014. The eligible population included residents over the age of 40 and had health medical records in community health centers. Community residents were recruited by responding to recruitment posters that detailed the study and inviting phone calls. The study design and selection criteria have been introduced before. $^{20}$ We used the cross-sectional data collected between May 2014 and July 2014 from the whole database. Initially, 3,882 participants were involved in the study. After excluding individuals with missing data on serum creatinine measurement and/or carotid artery ultrasonography, a total analytic sample of 3,815 remained. Ethical approval for this study was obtained from the ethics committee of Peking University First Hospital, and each participant provided written informed consent. We adhered to the principles of the Declaration of Helsinki.
The procedures followed were in accordance with institutional guidelines. ${ }^{20}$

\section{Data collection procedures}

The data were collected by trained research staff according to a standard operating procedure. After obtaining informed consent, trained interviewer administered a standard questionnaire that collected detailed information on demographics, lifestyle factors, and medical history. Physical exercise was defined as consciously doing exercise for $>30$ mins per time in the last 3 months. Current drinking habit was defined as drinking once per week at least half a year. Former drinking was defined as refraining from drinking for at least half a year. Current smoking was defined as smoking one cigarette per day for at least half a year, and a lower quantity was defined as non-smoking. Former smoking was defined as cessation of smoking for at least half a year.

Participants underwent a routine physical examination as well as blood sampling. Physical examination included standardized measurements of height and weight, and body mass index (BMI) was calculated. Venous blood samples were drawn from the antecubital vein with $4 \mathrm{~mL}$ coagulation-promoting vacuum tubes in the morning after an overnight fasting. All blood specimens were processed at the field centers and immediately frozen until they were shipped. Biochemistry index in plasma, including FBG, $2 \mathrm{hr}$ PG, total cholesterol (TC), total triglycerides (TG), low-density lipoprotein cholesterol (LDL-C), high-density lipoprotein cholesterol were obtained by the automatic biochemical analyzer (Roche-C8000). Hyperlipidemia was defined as LDL-C $\geq 3.37 \mathrm{mmol} / \mathrm{L}$ or $\mathrm{TG} \geq 1.7 \mathrm{mmol} /$ $\mathrm{L}$ or $\mathrm{TC} \geq 5.18 \mathrm{mmol} / \mathrm{L}$ or self-reported history of hyperlipidemia or use of lipid-lowering drugs.

\section{Definition of T2DM and hypertension}

All participants underwent standard 75-g oral glucose tolerance tests (or, T2DM patients consume equivalent bread) with 2 hrs plasma glucose sampled. In light of the WHO diagnostic criteria from 1999, participants were defined as having T2DM if they had an $F B G \geq 7.0 \mathrm{mmol} / \mathrm{L}$ and OGTT $\geq 11.1 \mathrm{mmol} / \mathrm{L}$ or self-reported history of T2DM or if they were currently taking any anti-T2DM treatment.

Seated peripheral (brachial) BP was obtained for each participant after a 5-min rest using an Omron HEM-7117 electronic sphygmomanometer with the standard method of calibration and appropriately sized cuffs. BP was measured three times on the right arm at the level of the heart 
with a gap of 1 min between consecutive measurements. The average of the three measurements was used as the final BP-value. According to 2013 ESC-ESH guidelines, hypertension was defined as $\mathrm{SBP} \geq 140 \mathrm{mmHg}$ or $\mathrm{DBP} \geq 90$ $\mathrm{mmHg}$, self-reported history of high blood pressure or antihypertensive drug usage.

\section{Estimated glomerular filtration rate (eGFR)}

Serum creatinine ( $\mu \mathrm{mol} / \mathrm{L})$ was measured using Jaffe kinetic method on a Hitachi 7180 Automatic Analyzer in the laboratory of Peking University First Hospital. eGFR was estimated using the following equation derived from the Chronic Kidney Disease Epidemiology Collaboration (CKD-EPI): ${ }^{21}$

$\mathrm{eGFR}=141 \times \min (\mathrm{SCr} / \kappa, 1) \alpha \times \max (\mathrm{SCr} / \kappa, 1)-1.209$ $\times 0.993$ Age $[\times 1.018$ if female $]$,

where Scr is serum creatinine concentration $(\mathrm{mg} / \mathrm{dL})$ and age in years; $\kappa, 0.7$ for females and 0.9 for males; $\alpha$, -0.329 for females and -0.411 for males; min, the minimum of Scr/ $k$ or 1 ; and max, the maximum of Scr/ $k$ or 1 . According to KDOQI Clinical Practice Guidelines for Chronic Kidney Disease 2015. ${ }^{22}$ We refer to an $\mathrm{eGFR}<60 \mathrm{~mL} / \mathrm{min} / 1.73 \mathrm{~m}^{2}$ as decreased GFR.

\section{Carotid ultrasonography}

All participants underwent carotid ultrasonography using the GE Vivid ultrasound system equipped with an $8-\mathrm{MHz}$ linear array vascular probe by a standardized protocol. In brief, carotid ultrasound was performed according to the standardized scanning and reading protocols by a trained and certified sonographer. Our Carotid Intima-Media Thickness (cIMT) protocol followed the Mannheim Carotid Intima-Media Thickness and Plaque Consensus (2004-2006-2011). ${ }^{23}$ The cIMT of both the left and right common carotid arteries (CCAs) was measured.

Plaques were defined as focal structures encroaching into the arterial lumen of at least $0.5 \mathrm{~mm}$ or $50 \%$ of the surrounding cIMT value, or demonstrating a thickness $>1.5 \mathrm{~mm}$ as measured from the intima-lumen interface to the media-adventitia interface, at levels of CCA, bifurcation, internal carotid artery and external carotid artery. ${ }^{23}$ Each plaque was measured in a longitudinal view, choosing the plane in which the plaque is maximal. The view chosen was frozen and magnified, the perimeter of the plaque was traced with a cursor on the screen, and the cross-sectional area of that plaque was measured. Sonographers measured the plaque area twice per plaque and then took the mean value. In this study, carotid plaque was characterized by its presence, number, and area. Number of carotid plaques was categorized into five groups: $0,1,2,3$ and $\geq 4$ according to the distribution. The sum of all plaque areas was defined as the total plaque area.

\section{Statistical analysis}

All data were represented as mean (SD) for continuous variables and proportion for categorical variables. The primary outcomes of interest included the presence, number, and total area of carotid plaque. The primary predictors were T2DM (dichotomous), hypertension (dichotomous) and eGFR that were evaluated as both dichotomous and continuous variable. First, we compared the demographic characteristics according to the presence of carotid plaques using Student's $t$-test for continuous variables, and Chi-square test for categorical ones. Next, we assessed the separate relation of T2DM, hypertension and eGFR (dichotomous and continuous variable) to the presence and number of carotid plaques using logistic regression model and multinomial logistic regression, respectively. We further used generalized linear regression to examine the associations between T2DM, hypertension, eGFR and the total area of plaque. To determine whether a joint effect exists between T2DM, hypertension and decreased GFR with respect to the characteristics of plaques, we performed stratified analyses according to diabetes and hypertension status, and tested multiplicative interactions by creating an interaction term and using a likelihood ratio test. Finally, we calculated the ORs associated with different combinations of T2DM, hypertension and decreased GFR.

Potential confounders were determined a priori; final models adjusted for the following covariates: age, sex, education level, BMI, drinking and smoking status, physical exercise, hypertension, hyperlipidemia, and diabetes and hypertension medications. ORs and their 95\% CIs were computed for primary predictors. Significance testing for all models was 2 -sided and a $P<0.05$ was considered statistically significant. All analyses were performed using SAS version 9.4 software (SAS Institute Inc., Cary, NC, USA).

\section{Results}

\section{Baseline characteristics categorized by the presence of carotid plaque}

Table 1 displays the characteristics of the study population according to the presence of carotid plaque. Of all 
Table I Characteristics of participants in the total sample and subgroups categorized by the presence of carotid plaque

\begin{tabular}{|c|c|c|c|c|}
\hline Characteristics & $\begin{array}{l}\text { Total sample } \\
(n=3,8 \mid 5)\end{array}$ & $\begin{array}{l}\text { Plaque absent } \\
(n=I, 65 I)\end{array}$ & $\begin{array}{l}\text { Plaque present } \\
(n=2,164)\end{array}$ & $\mathbf{P}$ \\
\hline Age, years, mean (SD) & $59.10(8.65)$ & $55.63(7.14)$ & $61.74(8.77)$ & $<0.001$ \\
\hline Male gender, N (\%) & I,377 (36.09) & $433(26.23)$ & $944(43.57)$ & $<0.001$ \\
\hline Body mass index, $\mathrm{kg} / \mathrm{m}^{2}$, mean (SD) & $25.98(3.54)$ & $25.85(3.57)$ & $26.08(3.5 I)$ & 0.0418 \\
\hline Education, N (\%) & & & & $<0.001$ \\
\hline$\leq$ Middle school & $\mathrm{I}, 460(38.27)$ & $500(30.28)$ & $960(44.36)$ & \\
\hline High school & I,732 (45.40) & $873(52.88)$ & $859(39.70)$ & \\
\hline$\geq$ Undergraduate & $623(16.33)$ & $278(16.84)$ & $345(15.94)$ & \\
\hline Physical exercise, $\geq 5$ times/per week, $N(\%)$ & 2,897 (75.94) & $1,210(73.29)$ & I,687 (77.96) & $<0.001$ \\
\hline Drinking status, $\mathrm{N}(\%)$ & & & & $<0.001$ \\
\hline Never & $3,211(84.17)$ & $\mathrm{I}, 444(87.46)$ & I,767 (8I.65) & \\
\hline Quit & $69(1.81)$ & $25(1.5 I)$ & $44(2.03)$ & \\
\hline Current & $535(14.02)$ & $182(11.02)$ & $353(16.31)$ & \\
\hline Smoking status, N (\%) & & & & $<0.001$ \\
\hline Never & $3,107(81.44)$ & $1,413(85.58)$ & $\mathrm{I}, 694(78.28)$ & \\
\hline Quit & $93(2.44)$ & $27(1.64)$ & $66(3.05)$ & \\
\hline Current & $615(16.12)$ & $211(12.78)$ & $404(18.67)$ & \\
\hline $\begin{array}{l}\text { Glomerular filtration rate, } \mathrm{mL} / \mathrm{min} \text { per } 1.73 \mathrm{~m}^{2} \text {, mean } \\
(\mathrm{SD})\end{array}$ & $69.60(11.69)$ & $71.75(11.07)$ & $67.95(11.88)$ & $<0.001$ \\
\hline Decreased GFR, N (\%) & $696(18.24)$ & $200(12.11)$ & $496(22.92)$ & $<0.001$ \\
\hline T2DM, N (\%) & $776(20.34)$ & $207(12.54)$ & $569(26.29)$ & $<0.001$ \\
\hline T2DM duration, years, mean (SD) & $9.25(7.60)$ & $7.81(6.62)$ & $9.74(7.85)$ & 0.006 \\
\hline Hypertension, N (\%) & I,782 (46.54) & $580(35.09)$ & $1,202(55.24)$ & $<0.001$ \\
\hline Hypertension duration, years, mean (SD) & I2.9| (10.77) & $10.86(9.48)$ & $13.86(11.20)$ & $<0.001$ \\
\hline Hyperlipidemia, N (\%) & $2,649(69.44)$ & $\mathrm{I}, 098(66.5 \mathrm{I})$ & I,55I (7I.67) & $<0.001$ \\
\hline Hypertension medication, N (\%) & $719(21.94)$ & $247(16.18)$ & $472(26.97)$ & $<0.001$ \\
\hline Diabetes medication, $\mathrm{N}$ (\%) & $237(7.23)$ & $65(4.26)$ & $172(9.83)$ & $<0.001$ \\
\hline Total cholesterol, mmol/L, mean (SD) & $5.00(0.99)$ & $5.04(0.95)$ & $4.97(1.01)$ & 0.0088 \\
\hline Triglyceride, mmol/L, mean (SD) & $1.75(1.36)$ & $1.77(1.53)$ & $1.74(1.21)$ & 0.4073 \\
\hline HDL-cholesterol, mmol/L, mean (SD) & $1.22(0.29)$ & $1.25(0.30)$ & $1.20(0.28)$ & $<0.001$ \\
\hline LDL-cholesterol, mmol/L, mean (SD) & $2.92(0.81)$ & $2.94(0.77)$ & $2.92(0.84)$ & 0.3558 \\
\hline \multicolumn{5}{|l|}{ The number of plaque, $\mathrm{N}(\%)$} \\
\hline 0 & I,65I (43.28) & $\mathrm{I}, 65 \mathrm{I}(100.00)$ & & \\
\hline 1 & 944 (24.74) & & 944 (43.62) & \\
\hline 2 & $592(15.52)$ & & $592(27.36)$ & \\
\hline
\end{tabular}


Table I (Continued).

\begin{tabular}{|c|c|c|c|c|}
\hline Characteristics & $\begin{array}{l}\text { Total sample } \\
(n=3,8 \mid 5)\end{array}$ & $\begin{array}{l}\text { Plaque absent } \\
(n=I, 65 I)\end{array}$ & $\begin{array}{l}\text { Plaque present } \\
(n=2,164)\end{array}$ & $P$ \\
\hline 3 & $257(6.74)$ & & $257($ (II.88) & \\
\hline 4 & 371 (9.72) & & $371(17.14)$ & \\
\hline
\end{tabular}

Abbreviations: T2DM, type 2 diabetes; eGFR, estimated glomerular filtration rate; HDL-C, high-density lipoprotein cholesterol; LDL-C low-density lipoprotein cholesterol; TC, total cholesterol; TG, total triglycerides.

the 3,815 individuals enrolled, mean (SD) age of participants was $59.10(8.65)$ years, $36.09 \%$ were male, and mean (SD) eGFR was $69.60(11.69) \mathrm{mL} / \mathrm{min} / 1.73 \mathrm{~m}^{2}$. A total of 2,164 participants $(56.72 \%)$ had carotid plaque, 776 (20.34\%) were with T2DM. Participants who developed carotid plaque tended to be older and to have a higher prevalence of decreased GFR, T2DM, hypertension, hyperlipidemia, and medications for hypertension or diabetes (all $P_{\mathbf{S}}<0.001$ ). There were also significant differences with regard to the distribution of education, drinking and smoking status, duration of diabetes and hypertension between two groups (all $\left.P_{\mathrm{S}}<0.001\right)$.

\section{T2DM, hypertension, eGFR, and carotid plaque}

The multivariable-adjusted relationships between T2DM, hypertension, eGFR (dichotomous and continuous variable) and carotid plaque among all the subjects are provided in Table 2. After adjusting for potential confounders, logistic regression analysis showed that OR of T2DM was 1.87 (95\% CI, 1.55-2.25) for an increased risk of carotid plaque. T2DM was also significantly related to plaque number (OR, $1.97 ; 95 \% \mathrm{CI}$, $1.70-2.28)$, and total plaque area $(\beta, 6.50 ; 95 \% \mathrm{CI}$, 2.93-10.08). Similarly, hypertension was independently associated with carotid plaque presence (OR, 1.33; 95\% CI, 1.10-1.59), number (OR, 1.50; 95\% CI, 1.28-1.76), and total area $(\beta, 6.52 ; 95 \% \mathrm{CI}, 3.57-9.47)$.

Significant correlations between kidney dysfunction and carotid plaque were also observed. As a dichotomous variable, decreased GFR was independently associated with presence (OR, 1.27; 95\% CI, 1.04-1.56), number $(\mathrm{OR}, 1.32 ; 95 \% \mathrm{CI}, 1.12-1.56)$, and total area $(\beta, 5.30$; $95 \%$ CI, 2.23-8.37) of carotid plaque. While as a continuous variable, eGFR had an OR of $0.99(95 \%$ CI, 0.98-0.99) for both the presence and number of carotid plaques and showed a negative correlation with total plaque area $(\beta,-0.22 ; 95 \% \mathrm{CI}-0.33-0.12)$.

Table 2 Association of T2DM, hypertension, and eGFR with carotid plaque

\begin{tabular}{|c|c|c|c|c|c|c|}
\hline \multirow[t]{2}{*}{ Diseases } & \multicolumn{2}{|c|}{ Carotid plaque presence } & \multicolumn{2}{|c|}{ Carotid plaque number } & \multicolumn{2}{|l|}{ Total plaque area } \\
\hline & OR $(95 \% \mathrm{Cl})$ & $P$ & OR $(95 \% \mathrm{Cl})$ & $\mathbf{P}$ & $\beta(95 \% \mathrm{Cl})$ & $P$ \\
\hline \multicolumn{7}{|l|}{ T2DM } \\
\hline No & 1.00 (Reference) & & I.00 (Reference) & & 0 (Reference) & \\
\hline Yes & $1.87(1.55-2.25)$ & $<0.001$ & $1.97(1.7-2.28)$ & $<0.001$ & $6.50(2.93-10.08)$ & $<0.001$ \\
\hline \multicolumn{7}{|l|}{ Hypertension } \\
\hline No & 1.00 (Reference) & & I.00 (Reference) & & 0 (Reference) & \\
\hline Yes & $1.33(1.1-1.59)$ & 0.008 & $1.5(1.28-1.76)$ & $<0.001$ & $6.52(3.57-9.47)$ & $<0.001$ \\
\hline \multicolumn{7}{|l|}{ Decreased GFR } \\
\hline No & 1.00 (Reference) & & I.00 (Reference) & & 0 (Reference) & \\
\hline Yes & $1.27(1.04-1.56)$ & 0.021 & $1.32(1.12-1.56)$ & $<0.001$ & $5.30(2.23-8.37)$ & $<0.001$ \\
\hline eGFR per unit increase & $0.99(0.98-0.99)$ & $<0.001$ & $0.99(0.98-0.99)$ & $<0.001$ & $-0.22(-0.33$ to -0.12$)$ & $<0.001$ \\
\hline
\end{tabular}

Notes: Decreased GFR is defined as a GFR $<60 \mathrm{~mL} / \mathrm{min} / \mathrm{I} .73 \mathrm{~m}^{2}$; logistic regression models, multinomial logistic regression, and generalized linear regression models were adjusted for the confounders including age, sex, education, BMI, drinking and smoking status, physical exercise, hypertension, hyperlipidemia, and diabetes and hypertension medications. Abbeviations: T2DM, type 2 diabetes; eGFR, estimated glomerular filtration rate; BMI, body mass index. 


\section{Association of eGFR and T2DM or hypertension with carotid plaque}

We observed important differences in the association between eGFR and carotid plaque across the strata for T2DM (Table 3). In the absence of T2DM, changes in eGFR were associated with increased presence, number and total area of carotid plaques (all $P_{\mathbf{S}}<0.05$ ). However, in the presence of T2DM, although eGFR per unit increase was still associated with $0.27(95 \% \mathrm{CI}-0.50$ to -0.03$) \mathrm{mm}^{2}$ decrease in total plaque area, the associations of eGFR with the presence and number of plaques lost their significance. Nevertheless, multiplicative interactions between T2DM and eGFR were not significant (all $P \mathrm{~s}>0.05$ ). In contrast, stratified analysis did not identify effect modification of hypertension to eGFR change and carotid plaque (Table 4). T2DM and eGFR are significantly associated with the presence, number and total area of plaques among participants both with and without hypertension.

We further explored the joint association of decreased GFR, T2DM, and hypertension with carotid plaque (Table 5). Participants without any one of these three diseases were used as the reference. For the presence of carotid plaque, the OR of a combination of T2DM, hypertension and decreased GFR (OR, 2.33; 95\% CI, 1.49-3.64), although slightly less than that of T2DM combined with hypertension, was obviously greater than that of T2DM, hypertension or decreased GFR alone. For number of carotid plaques, the OR was greatest when T2DM, hypertension and decreased GFR were present (OR, 3.69; 95\% CI, 2.65-5.14). In the absence of hypertension, decreased GFR was not significantly associated with increased total plaque area in T2DM patients $(\beta, 7.20 ; 95 \% \mathrm{CI},-2.78-17.18)$. The total area of carotid plaque increased for hypertension patients in the presence of either decreased GFR ( $\beta$, $11.30 ; 95 \% \mathrm{CI}, 6.38-16.21)$ or T2DM $(\beta, 13.00 ; 95 \%$ CI, 8.00-18.00). The strongest association was also seen in the combination of three status $(\beta, 20.63 ; 95 \% \mathrm{CI}$, 14.04-27.22).

\section{Discussion}

In this study, we found that the association between decreased GFR and carotid plaque was influenced by T2DM and hypertension. A synergistic association was found between decreased GFR with T2DM or hypertension and the prevalence and severity of carotid plaque. Therefore, coexisting T2DM, hypertension and decreased GFR may contribute to further elevated risk of carotid plaque and CVD.

Multiple studies have been carried out to assess the association of different cutoffs of eGFR with the risk of CVD after adjustment for traditional risk factors. ${ }^{24-27}$ Carotid plaque represents very high CVD risk that generally requires the preventive use of CVD drugs, ${ }^{28,29}$ whereas the association between eGFR and carotid plaque may seem paradoxical. In Beijing Longitudinal Study of Aging (BLSA), eGFR was related to the presence of carotid plaque. ${ }^{30}$ Similar results were found in the Third

Table 3 Association between eGFR and carotid plaque in different sub-groups according to the presence or not of T2DM

\begin{tabular}{|c|c|c|c|c|c|c|}
\hline \multirow[t]{2}{*}{ Diseases } & \multicolumn{2}{|c|}{ Carotid plaque presence } & \multicolumn{2}{|c|}{ Carotid plaque number } & \multicolumn{2}{|l|}{ Total plaque area } \\
\hline & OR $(95 \% \mathrm{Cl})$ & $P$ & OR $(95 \% \mathrm{Cl})$ & $P$ & $\beta(95 \% \mathrm{Cl})$ & $P$ \\
\hline Non-diabetes & & & & & & \\
\hline $\begin{array}{l}\text { Decreased GFR } \\
\text { No } \\
\text { Yes } \\
\text { eGFR per unit increase }\end{array}$ & $\begin{array}{l}\text { I (Reference) } \\
\text { I.42(I.I2-I.78) } \\
0.99(0.98-0.99) \\
\end{array}$ & $\begin{array}{l}0.003 \\
<0.001\end{array}$ & $\begin{array}{l}\text { I (Reference) } \\
\text { I.38 (I.14-I.67) } \\
0.99(0.98-0.99) \\
\end{array}$ & $\begin{array}{l}<0.001 \\
<0.001 \\
\end{array}$ & $\begin{array}{l}0 \text { (Reference) } \\
5.35(1.83-8.86) \\
-0.21(-0.32 \text { to }-0.09)\end{array}$ & $\begin{array}{l}0.003 \\
<0.001\end{array}$ \\
\hline Diabetes & & & & & & \\
\hline $\begin{array}{l}\text { Decreased GFR } \\
\text { No } \\
\text { Yes } \\
\text { eGFR per unit increase }\end{array}$ & $\begin{array}{l}\text { I (Reference) } \\
0.82(0.52-1.29) \\
1.00(0.98-1.01)\end{array}$ & $\begin{array}{l}0.39 \\
0.55\end{array}$ & $\begin{array}{l}\text { I (Reference) } \\
\text { I.5। }(0.84-1.58) \\
0.99(0.98-1.00)\end{array}$ & $\begin{array}{l}0.39 \\
0.078\end{array}$ & $\begin{array}{l}0 \text { (Reference) } \\
5.26(-1.05-1 \mathrm{I} .58) \\
-0.27(-0.50 \text { to }-0.03)\end{array}$ & $\begin{array}{l}0.10 \\
0.027\end{array}$ \\
\hline
\end{tabular}

Notes: Decreased GFR is defined as a GFR $<60 \mathrm{~mL} / \mathrm{min} / 1.73 \mathrm{~m}^{2}$; logistic regression models, multinomial logistic regression, and generalized linear regression models were adjusted for the confounders including age, sex, education, BMI, drinking and smoking status, physical exercise, hypertension, hyperlipidemia, and diabetes and hypertension medications.

Abbreviations: T2DM, type2 diabetes; eGFR, estimated glomerular filtration rate; BMI, body mass index. 
Table 4 Association between eGFR and carotid plaque in different sub-groups according to the presence or not of hypertension

\begin{tabular}{|c|c|c|c|c|c|c|}
\hline \multirow[t]{2}{*}{ Diseases } & \multicolumn{2}{|c|}{ Carotid plaque presence } & \multicolumn{2}{|c|}{ Carotid plaque number } & \multicolumn{2}{|l|}{ Total plaque area } \\
\hline & OR $(95 \% \mathrm{Cl})$ & $P$ & OR (95\% Cl) & $P$ & $\beta(95 \% \mathrm{Cl})$ & $P$ \\
\hline Non-hypertension & & & & & & \\
\hline $\begin{array}{l}\text { Decreased GFR } \\
\text { No } \\
\text { Yes } \\
\text { eGFR per unit increase }\end{array}$ & $\begin{array}{l}\text { I (Reference) } \\
\text { I.35 (I.03-I.78) } \\
0.98(0.97-0.99)\end{array}$ & $\begin{array}{l}0.032 \\
<0.001\end{array}$ & $\begin{array}{l}\text { I (Reference) } \\
\text { I.25 (I.0I-I.54) } \\
0.98(0.97-0.99)\end{array}$ & $\begin{array}{l}0.011 \\
<0.001\end{array}$ & $\begin{array}{l}0 \text { (Reference) } \\
4.82(1.95-7.70) \\
-0.19(-0.28 \text { to }-0.10)\end{array}$ & $\begin{array}{l}0.001 \\
<0.001\end{array}$ \\
\hline Hypertension & & & & & & \\
\hline $\begin{array}{l}\text { Decreased GFR } \\
\text { No } \\
\text { Yes } \\
\text { eGFR per unit increase }\end{array}$ & $\begin{array}{l}\text { I (Reference) } \\
\text { I.22 (0.93-I.6I) } \\
0.99(0.98-0.99)\end{array}$ & $\begin{array}{l}0.16 \\
0.042\end{array}$ & $\begin{array}{l}\text { I (Reference) } \\
\text { I.25 (I.0I-I.54) } \\
0.99(0.98-0.99)\end{array}$ & $\begin{array}{l}0.039 \\
0.009\end{array}$ & $\begin{array}{l}0 \text { (Reference) } \\
6.04(0.79-11.29) \\
-0.26(-0.45 \text { to }-0.07)\end{array}$ & $\begin{array}{l}0.024 \\
0.008\end{array}$ \\
\hline
\end{tabular}

Notes: Decreased GFR is defined as a GFR $<60 \mathrm{~mL} / \mathrm{min} / 1.73 \mathrm{~m}^{2}$; logistic regression models, multinomial logistic regression, and generalized linear regression models were adjusted for the confounders including age, sex, education, BMI, drinking and smoking status, physical exercise, hypertension, hyperlipidemia, and diabetes and hypertension medications.

Abbreviations: T2DM, type 2 diabetes; eGFR, estimated glomerular filtration rate; BMI, body mass index.

National Health and Nutrition Examination Survey, for a significant association between low eGFR and plaque presence. $^{31}$ In contrast, in the Renal Iohexol Clearance Survey in Tromsø 6 (RENIS-T6), there was a tendency for a U-shaped relationship between eGFR and total plaque area, but nonlinear models were not statistically significant. ${ }^{32}$ A cross-sectional study on Japanese women reported that albuminuria, but not low eGFR, was associated with carotid plaque. ${ }^{33}$ Notably, most of these studies are hospital-based design and eGFR was assessed by different equations.

The present study was based on a large populationbased cohort with a relatively high proportion of participant having normal kidney function, while eGFR was estimated by the CKD-EPI equation. Our study revealed that decreased GFR was associated with the presence, number and total area of carotid plaque independent of conventional risk factors. Moreover, the relation of continuous form of eGFR to plaque indicated that even small reductions in GFR within the apparently normal range may contribute to the increased prevalence of plaque; thus, eGFR level may play a predictive role for cardiovascular events.

Although after adjusting for other covariates, decreased GFR was not significantly associated with the presence and number of carotid plaque in T2DM patients. This result is consistent with a previous study in which reduced eGFR was not associated with atherosclerosis in patients with T2DM. ${ }^{34}$ The result may be due to the fact that T2DM itself has such a powerful influence on carotid plaque that any effect of eGFR decline is masked. ${ }^{34}$

T2DM and hypertension are conventional risk factors for CVD. Affecting the entire vascular system, it is perhaps not surprising that T2DM is also associated with subclinical vascular disease measures. Multi-Ethnic Study of Atherosclerosis demonstrated that diabetes was associated with an increased prevalence of aortic valve calcium (incidence rate ratio: 2.1 for diabetes in women and 1.7 for men) and thoracic aortic calcification. ${ }^{35}$ Another population-based study in Iceland reported that T2DM was one of the strongest risk factors associated with the presence of carotid plaque (OR, 1.44; 95\% CI, 1.02-2.04). ${ }^{18}$ Similarly, it has long been established that systolic blood pressure (OR 1.2 per $10 \mathrm{mmHg}$ increase) is one of the predictors of carotid stenosis, ${ }^{36}$ with many studies confirming the direct correlation between hypertension and carotid plaque. ${ }^{37-39}$ In this study, the risk estimate that we observed for T2DM (OR, 1.87; 95\% CI, 1.55-2.25) and hypertension (OR, 1.33; 95\% CI, $1.10-1.59)$ is consistent with most previously reported estimates. ${ }^{18,40-42}$ The consistency of our findings with prior results lends weight to the evidence that T2DM and hypertension are strong detrimental factors for carotid plaque.

Although the relationship between individual risk factors and carotid plaque has been proved, the combined effect of three risk factors cannot be ignored. In this study, we found that OR for patients with 3 risk factors versus no risk factors for the number of plaques was 3.69 


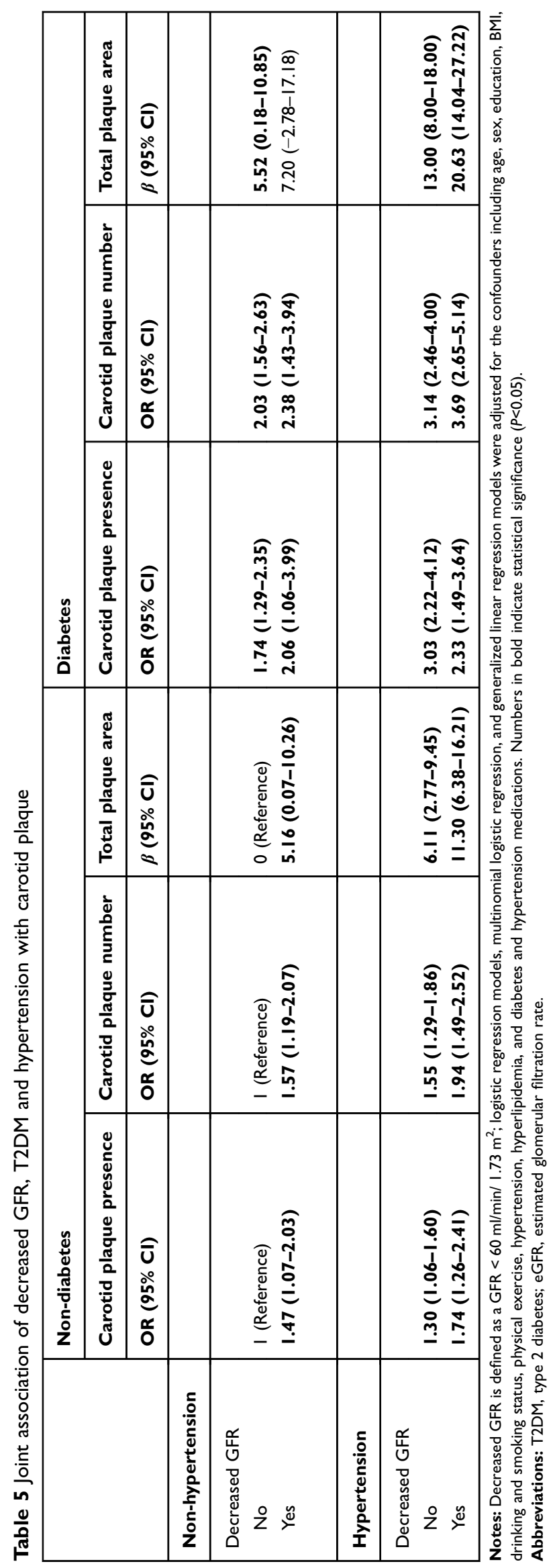


(95\% CI, 2.65-5.14). Chuang et al concluded that hypertension and dyslipidemia with type 2 diabetes have cumulative effects on the burden of carotid plaque. ${ }^{43}$ Niu et al in their comparative study concluded that three or more cardiovascular risk factors have a greater impact on cIMT and elasticity than two or less cardiovascular risk factors. ${ }^{44}$ Previous studies have shown that hypertension, T2DM, and CKD induce abnormal shearing pressure to the endothelium, inflammation, insulin resistance, endothelial dysfunction and more severe oxidative stress in blood, which all participate in the pathogenesis of vascular disease. ${ }^{43,45-47}$ However, the mechanisms by which three risk factors may have synergistic effects on the increased carotid plaque burden need to be confirmed in future studies.

Therefore, our study suggests that onset of nephropathy in patients with T2DM and hypertension increases the carotid plaque burden, while control of renal function could mitigate the adverse effects of T2DM and hypertension on carotid plaque progression. It is demonstrated that eGFR per unit increase was associated with 0.27 and 0.26 unit reduction in total plaque area in participants with T2DM and hypertension, respectively.

\section{Limitations}

Several limitations should be acknowledged. This is a cross-sectional study showing only associations between T2DM, hypertension, eGFR and carotid plaque, which limits the power of causal inference. In addition, our findings might be only generalizable to community-dwelling Chinese adults who would have been healthy enough to attend the health center assessment. However, our findings provide further evidence that T2DM, hypertension and kidney dysfunction are all important factors affecting subclinical CVD. More prospective, population-based studies with large cohort are needed to provide a better interpretation.

\section{Conclusion}

Individuals with coexisting decreased GFR, T2DM and hypertension are at high risk of carotid plaque, with each condition contributing additively to plaque risk. Additional studies are needed to determine the extent to which antidiabetic and anti-hypertensive therapy combined with the appropriate CKD treatment can lead to prevention of carotid atherosclerosis.

\section{Acknowledgments}

The study was supported by grants from the National Natural Science Foundation of China (81172768, 81872692), Beijing Municipal Natural Science Foundation (7182085) and the University of Michigan Health System and Peking University Health Science Center (UM-PUHSC) Joint Institute for Translational and Clinical Research (BMU20110177). We appreciate the staff in Pingguoyuan and Gucheng Community Health Centers and research coordinators for the assistance to data acquisition.

\section{Disclosure}

The authors report no conflicts of interest in this work.

\section{References}

1. Polak JF, Pencina MJ, Pencina KM, et al. Carotidwall intima-media thickness and cardiovascular events. $N$ Engl J Med. 2011;365:213-221. doi:10.1056/NEJMoa0910383

2. Chambless LE, Folsom AR, Clegg LX, et al. Carotid wall thickness is predictive of incident clinical stroke: the Atherosclerosis Risk in Communities (ARIC) study. Am J Epidemiol. 2000;151:478-487. doi:10.1093/oxfordjournals.aje.a010213

3. Chambless LE, Heiss G, Folsom AR, et al. Association of coronary heart disease incidence with carotid arterial wall thickness and major risk factors: the Atherosclerosis Risk in Communities (ARIC) study, 1987-1993. Am J Epidemiol. 1997;146:483-494. doi:10.1093/ oxfordjournals.aje.a009302

4. O'Leary DH, Polak JF, Kronmal RA, et al. Carotid-artery intima and media thickness as a risk factor for myocardial infarction and stroke in older adults Cardiovascular Health Study Collaborative Research Group. $N$ Engl J Med. 1999;340:14-22. doi:10.1056/NEJM199 905133401909

5. Nambi V, Chambless L, Folsom AR, et al. Carotid intima-media thickness and presence or absence of plaque improves prediction of coronary heart disease risk: the ARIC study. J Am Coll Cardiol. 2010;55:1600-1607. doi:10.1016/j.jacc.2009.11.075

6. Mirzabeigi P, Mohammadpour AH, Salarifar M, et al. The effect of curcumin on some of traditional and non-traditional cardiovascular risk factors: a pilot randomized, double-blind, placebo-controlled trial. Iran J Pharm Res. 2015;14(2):479-486.

7. Kottgen A, Russell SD, Loehr LR, et al. Reduced kidney function as a risk factor for incident heart failure: the Atherosclerosis Risk In Communities (ARIC) study. J Am Soc Nephrol. 2007;18: 1307-1315.

8. Abramson JL, Jurkovitz CT, Vaccarino V, Weintraub WS, McClellan W. Chronic kidney disease, anemia, and incident stroke in a middle-aged, community-based population: the ARIC study. Kidney Int. 2003;64:610-615. doi:10.1046/j.1523-1755.2003.00109.x

9. Astor BC, Coresh J, Heiss G, Pettitt D, Sarnak MJ. Kidney function and anemia as risk factors for coronary heart disease and mortality: the Atherosclerosis Risk in Communities (ARIC) study. Am Heart J. 2006;151:492-500. doi:10.1016/j.ahj.2005.10.019

10. Alonso A, Lopez FL, Matsushita K, et al. Chronic kidney disease is associated with the incidence of atrial fibrillation: the Atherosclerosis Risk In Communities (ARIC) study. Circulation. 2011;123:2946-2953. doi:10.1161/CIRCULATIONAHA.111.020982

11. Tanaka M, Abe Y, Furukado S, et al. Chronic kidney disease and carotid atherosclerosis. J Stroke Cerebrovascular Dis. 2012;21:47-51. doi:10.1016/j.jstrokecerebrovasdis.2010.03.018 
12. De CS, Rossi MC, Pellegrini F, et al. Kidney dysfunction and related cardiovascular risk factors among patients with type 2 diabetes. Nephrol Dialysis Transplant. 2014;29(3):657. doi:10. 1093/ndt/gft506

13. Pavkov ME, Knowler WC, Lemley KV, et al. Early renal function decline in type 2 diabetes. Clin J Am Soc Nephrol CJASN. 2012;7 (1):78. doi:10.2215/CJN.10251011

14. Bailey RA, Wang Y, Zhu V, et al. Chronic kidney disease in US adults with type 2 diabetes: an updated national estimate of prevalence based on kidney disease: improving Global Outcomes (KDIGO) staging. $B M C$ Res Notes. 2014;7(1):1-7. doi:10.1186/1756-0500-7-1

15. Verma A, Vyas S, Agarwal A, et al. Diabetic kidney disease and hypertension: a true love story. J Clin Diagn Res Jcdr. 2016;10(3):OC11.

16. Gray SP, Jandeleitdahm K. The pathobiology of diabetic vascular complications-cardiovascular and kidney disease. J Mol Med. 2014;92(5):441-452. doi:10.1007/s00109-014-1146-1

17. Högberg D, Kragsterman B, Björck M, Tjärnström J, Wanhainen A. Carotid artery atherosclerosis among 65-year-old Swedish men a population-based screening study. Eur J Vasc Endovascular Surg. 2014;48(1):5-10. doi:10.1016/j.ejvs.2014.02.004

18. Ran S, Aspelund T, Bjornsdottir G, et al. Prevalence and determinants of carotid plaque in the cross-sectional REFINE-Reykjavik study. BMJ Open. 2016;6(11):e012457. doi:10.1136/bmjopen-2016012457

19. Mostaza JM, Lahoz C, Salinero-Fort MA, et al. Carotid atherosclerosis severity in relation to glycemic status: a cross-sectional population study. Atherosclerosis. 2015;242(2):377-382. doi:10.1016/j. atherosclerosis.2015.07.028

20. Fan F, Qi L, Jia J, et al. Noninvasive central systolic blood pressure is more strongly related to kidney function decline than peripheral systolic blood pressure in a Chinese community-based population. Hypertension. 2016;67:1166-1172. doi:10.1161/HYPERTENSIO NAHA.115.07019

21. Levey AS, Stevens LA, Schmid CH, et al. CKD-EPI (Chronic kidney disease epidemiology collaboration). A new equationto estimate glomerular fltration rate. Ann Intern Med. 2009; 150:604-612.

22. Andrassy KM. Comments on 'KDIGO 2012 clinical practice guideline for the evaluation and management of chronic kidney disease'. Kidney Int. 2013;84(3):622-623. doi:10.1038/ ki.2013.243

23. Touboul PJ, Hennerici MG, Meairs S, et al. Mannheim carotid intima-media thickness and plaque consensus (2004-20062011). Cerebrovasc Dis. 2012;34:290-296. doi:10.1159/000343145

24. Go AS, Chertow GM, Fan D, et al. Chronic kidney disease and the risks of death, cardiovascular events, and hospitalization. $N \mathrm{Engl}$ $J$ Med. 2004;351(13):1296-1305. doi:10.1056/NEJMoa041031

25. Shlipak MG, Fried LF, Crump C, et al. Cardiovascular disease risk status in elderly persons with renal insufficiency. Kidney Int. 2002;62:997-1004. doi:10.1046/j.1523-1755.2002.00522.x

26. Muntner P, He J, Hamm L, Loria C, Whelton PK. Renal insufficiency and subsequent death resulting from cardiovascular disease in the United States. J Am Soc Nephrol. 2002;13:745-753.

27. Ninomiya T, Kiyohara Y, Tokuda Y, et al. Impact of kidney disease and blood pressure on the development of cardiovascular disease: an overview from the Japan Arteriosclerosis Longitudinal study. Circulation. 2008;118(25):2694-2701. doi:10.1161/CIRCULAT IONAHA.108.792903

28. Perk J, De Backer G, Gohlke H, et al. European guidelines on cardiovascular disease prevention in clinical practice (version 2012). The Fifth Joint Task Force of the European Society of Cardiology and other Societies on cardiovascular disease prevention in clinical practice (constituted by representatives of nine societies and by invited experts). Developed with the special contribution of the European Association for Cardiovascular Prevention \& Rehabilitation (EACPR). Atherosclerosis. 2012;223:1-68.
29. Greenland P, Alpert JS, Beller GA, et al. 2010 ACCF/AHA guideline for assessment of cardiovascular risk in asymptomatic adults: a report of the American College of Cardiology Foundation/American Heart Association Task Force on practice guidelines. J Am Coll Cardiol. 2010;56:e50-103. doi:10.1016/j.jacc.2010.09.001

30. Gu X, Fang X, Hua Y, et al. Association between kidney dysfunction and carotid atherosclerosis in community-based older adults in China. Angiology. 2015;67(3):252-258.

31. Leoncini G, Viazzi F, Parodi D, et al. Mild renal dysfunction and cardiovascular risk in hypertensive patients. J Am Soc Nephrol Jasn. 2004;15 Suppl 1(1):S88. doi:10.1097/01.ASN.0000093245.78097.CD

32. Eriksen BO, Løchen ML, Arntzen KA, et al. Subclinical cardiovascular disease is associated with a high glomerular filtration rate in the nondiabetic general population. Kidney International. 2014;86 (1):146-153. doi:10.1038/ki.2013.470

33. Ishizaka Y, Ishizaka N, Tani M, et al. Relationship between albuminuria, low eGFR, and carotid atherosclerosis in Japanese Women. Kidney Blood Press Res. 2008;31(3):164-170. doi:10.1159/000131750

34. Sjöblom P, Nystrom FH, Länne T, et al. Microalbuminuria, but not reduced eGFR, is associated with cardiovascular subclinical organ damage in type 2 diabetes. Diabetes Metab. 2014;40(1):49-55. doi:10.1016/j.diabet.2013.09.008

35. Katz R, Wong ND, Kronmal R, et al. Features of the metabolic syndrome and diabetes mellitus as predictors of aortic valve calcification in the multi-ethnic study of atherosclerosis. Circulation. 2006;113:2113-2119. doi:10.1161/CIRCULATIONAHA.105.598086

36. Fine-Edelstein JS, Wolf PA, O'Leary DH, et al. Precursors of extracranial carotid atherosclerosis in the Framingham Study. Neurology. 1994;44(6):1046-1050. doi:10.1212/wnl.44.6.1046

37. Cao JJ, Thach C, Manolio TA, et al. Association of carotid artery intima-media thickness, plaques, and C-reactive protein with future cardiovascular disease and all-cause mortality: the Cardiovascular Health study. Circulation. 2007;116:32-38. doi:10.1161/ CIRCULATIONAHA.106.645606

38. Spence JD, Hegele RA. Noninvasive phenotypes of atherosclerosis: similar windows but different views. Stroke. 2004;35:649-653. doi:10.1161/01.STR.0000116103.19029.DB

39. Delcker A, Diener HC, Wilhelm H. Influence of vascular risk factors for atherosclerotic carotid artery plaque progression. Stroke. 1995;26:2016-2022. doi:10.1161/01.STR.26.11.2016

40. Prati P, Vanuzzo D, Casaroli M, et al. Prevalence and determinants of carotid atherosclerosis in a general population. Stroke. 1992;23 (12):1705. doi:10.1161/01.STR.23.12.1705

41. Zhan C, Shi M, Yang Y, et al. Prevalence and risk factors of carotid plaque among middle-aged and elderly adults in Rural Tianjin, China. Sci Rep. 2016;6:23870. doi:10.1038/srep23870

42. Huang LC, Lin RT, Chen CF, et al. Predictors of carotid intima-media thickness and plaque progression in a Chinese Population. J Atherosclerosis Thrombosis. 2016. doi:10.5551/jat.32177

43. Yuan C, Lai C, Chan L, et al. Cumulative effects of hypertension, dyslipidemia, and chronic kidney disease on carotid atherosclerosis in Chinese patients with type 2 diabetes mellitus. J Diabetes Res. 2014;2014(19):179686. doi:10.1155/2014/179686

44. Niu L, Zhang Y, Qian M, et al. Impact of multiple cardiovascular risk factors on carotid intima-media thickness and elasticity. PLoS One. 2013;8:e67809. doi:10.1371/journal.pone.0067809

45. Manjunath G, Tighiouart H, Ibrahim H, et al. Level of kidney function as a risk factor for atherosclerotic cardiovascular disease in the community. J Am Coll Cardiol. 2003;41:47-55. doi:10.1016/S0735-1097(02)02663-3

46. Sonmez A, Yilmaz MI, Saglam M, et al. The relationship between hemoglobin levels and endothelial functions in diabetes mellitus. Clin J Am Soc Nephrol CJASN. 2010;5(1):45-50. doi:10.2215/CJN.05080709

47. Nobuo K, Uchida HA, Isao S, et al. Chronic kidney disease is associated with carotid atherosclerosis and symptomatic ischaemic stroke. J Int Med. 2018;46(9):3873-3883. doi:10.1177/ 0300060518781619 


\section{Publish your work in this journal}

Diabetes, Metabolic Syndrome and Obesity: Targets and Therapy is an international, peer-reviewed open-access journal committed to the rapid publication of the latest laboratory and clinical findings in the fields of diabetes, metabolic syndrome and obesity research. Original research, review, case reports, hypothesis formation, expert opinion and commentaries are all considered for publication. The manuscript management system is completely online and includes a very quick and fair peer-review system, which is all easy to use. Visit http://www.dovepress.com/testimonials.php to read real quotes from published authors. 